\title{
OPEN A modulation-doped
}

\section{heterostructure-based terahertz photoconductive antenna emitter with recessed metal contacts}

\author{
Jessica Afalla $a^{1,2,5}$, Alexander De Los Reyes ${ }^{3,5}$, Neil Irvin Cabello ${ }^{3,5}$, Victor DC Andres Vistro ${ }^{3 凶,}$ \\ Maria Angela Faustino ${ }^{4}$, John Paul Ferrolino ${ }^{4}$, Elizabeth Ann Prieto ${ }^{4}$, Hannah Bardolaza ${ }^{3}$, \\ Gerald Angelo R. Catindig ${ }^{3}$, Karl Cedric Gonzales ${ }^{3}$, Valynn Katrine Mag-usara ${ }^{2}$, \\ Hideaki Kitahara ${ }^{2}$, Armando S. Somintac ${ }^{3}$, Arnel A. Salvador ${ }^{3}$, Masahiko Tani ${ }^{2}$ \& \\ Elmer S. Estacio ${ }^{3 凶}$
}

We present the implementation of an efficient terahertz (THz) photoconductive antenna (PCA) emitter design that utilizes high mobility carriers in the two-dimensional electron gas (2DEG) of a modulationdoped heterostructure (MDH). The PCA design is fabricated with recessed metal electrodes in direct contact with the 2DEG region of the MDH. We compare the performance of the MDH PCA having recessed contacts with a PCA fabricated on bulk semi-insulating GaAs, on low temperature-grown GaAs, and a MDH PCA with the contacts fabricated on the surface. By recessing the contacts, the applied bias can effectively accelerate the high-mobility carriers within the 2DEG, which increases the $\mathrm{THz}$ power emission by at least an order of magnitude compared to those with conventional structures. The dynamic range $(62 \mathrm{~dB})$ and bandwidth characteristics $(3.2 \mathrm{THz})$ in the power spectrum are shown to be comparable with the reference samples. Drude-Lorentz simulations corroborate the results that the higher-mobility carriers in the $\mathrm{MDH}$, increase the THz emission. The saturation characteristics were also measured via optical fluence dependence, revealing a lower saturation value compared to the reference samples. The high THz conversion efficiency of the MDH-PCA with recessed contacts at low optical power makes it an attractive candidate for THz-time domain spectroscopy systems powered by low power fiber lasers.

The terahertz (THz) band refers to the $100 \mathrm{GHz}-30 \mathrm{THz}$ frequencies of the electromagnetic spectrum ${ }^{1}$. The applications of $\mathrm{THz}$ radiation in numerous fields, such as energy ${ }^{2}$, medicine ${ }^{3}$, manufacturing ${ }^{4}$, imaging ${ }^{5}$ and $_{\text {arts }}^{6}$, have driven the demand for more intense $\mathrm{THz}$ sources. One of the most popularly-used broadband $\mathrm{THz}$ sources for spectroscopic applications is the photoconductive antenna (PCA $)^{7}$. It is typically composed of a semiconductor substrate material having two surface metal electrodes, with the electrodes separated by micrometer scale, forming the photoconductive gap. In its typical operation, the gap of the PCA THz emitter is biased with either a dc or ac voltage and illuminated with ultrafast laser pulses. Upon illumination, electron-hole pairs are photogenerated in the semiconductor substrate. As the electrons and holes drift in opposite directions towards the biased metal electrodes, they form a transient current, which emits $\mathrm{THz}$ radiation via the metal contacts functioning as an antenna ${ }^{8}$. The performance of the PCA is dictated by the characteristics of the photoconductive substrate, such as carrier lifetime, band gap, breakdown voltage, resistivity and electron mobility ${ }^{8}$. Low temperature-grown gallium arsenide (LT-GaAs) is among the most commonly used PCA substrate material due to its short carrier lifetime and its high breakdown-voltage characteristics, despite LT-GaAs having lower electron mobility compared to crystalline GaAs ${ }^{9}$. Meanwhile, semi-insulating (SI) GaAs PCAs have also been commercially utilized as $\mathrm{THz}$ emitters due instead to their high mobilities ${ }^{10}$. Following this approach, a more intense $\mathrm{THz}$ emission can be

\footnotetext{
${ }^{1}$ Graduate School of Pure and Applied Sciences, University of Tsukuba, Tsukuba 305-8573, Japan. ${ }^{2}$ Research Center for Development of Far Infrared Region, University of Fukui, Fukui 910-8507, Japan. ${ }^{3}$ National Institute of Physics, University of the Philippines Diliman, 1101 Quezon City, Philippines. ${ }^{4}$ Material Science and Engineering Program, University of the Philippines Diliman, 1101 Quezon City, Philippines. ${ }^{5}$ These authors contributed equally: Jessica Afalla, Alexander De Los Reyes and Neil Irvin Cabello. ${ }^{\square}$ email: dvistro@nip.upd.edu.ph; eestacio@nip.upd.edu.ph
} 


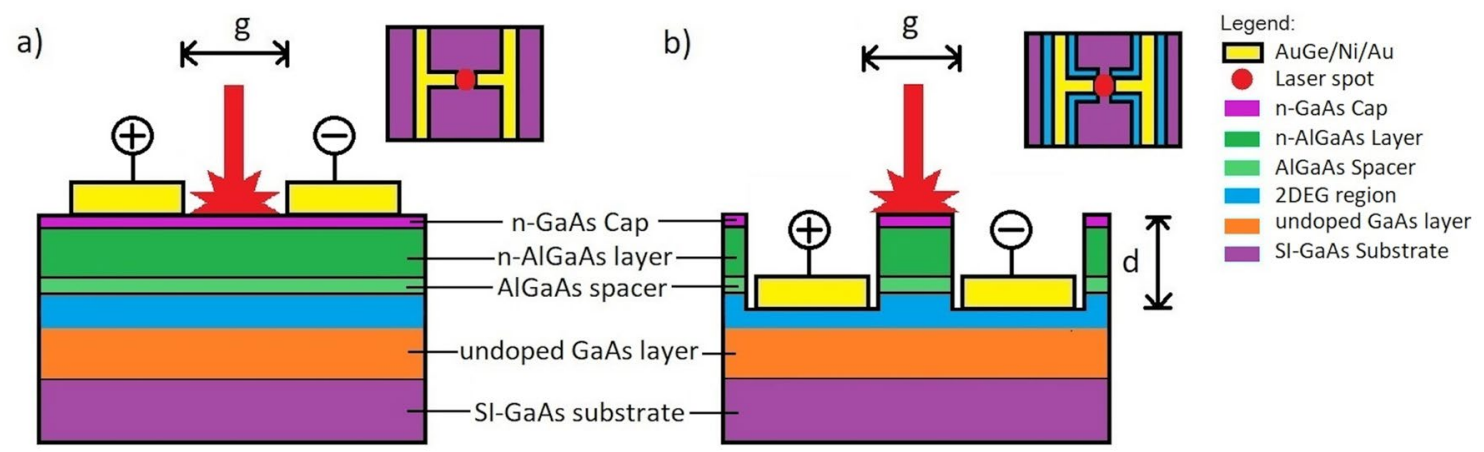

Figure 1. Schematic diagrams of the cross section of the MDH PCAs when the metal contacts are (a) at the surface of the $\mathrm{MDH}$ and (b) recessed. $g$ denotes the gap width, and $d$ denotes the etch depth. The inset figures show the top view of each of the MDH PCA. The diagrams are not to scale.

obtained from a PCA emitter that utilizes a GaAs-based, high electron mobility heterostructure; an example of which is the aluminum gallium arsenide ( $\mathrm{AlGaAs}$ )/GaAs modulation-doped heterostructure (MDH).

In a conventional AlGaAs/GaAs $\mathrm{MDH}, \mathrm{n}$-doped AlGaAs is separated from undoped GaAs by a thin spacer layer (Fig. 1a). Due to the alignment of the Fermi levels, a triangular well is formed in the conduction band. The confined carriers in this triangular well form a two-dimensional electron gas (2DEG) region, where the electrons have higher mobility and lower scattering from ionized impurities, compared to electrons in the bulk GaAs region ${ }^{11}$. Owing to the enhanced carrier mobility, the $\mathrm{MDH}$ is conventionally used for high-speed devices; particularly as a modulation-doped field effect transistor (MODFET), also referred to as "high electron mobility transistor (HEMT)", as well as applications in spectroscopy ${ }^{12}$, and optoelectronics ${ }^{13}$. The MDH utilized as a HEMT has been previously shown by Dyakonov et al. to function in the $\mathrm{THz}$ range as a detector, mixer, and multiplier owing to the utilization of the $2 \mathrm{DEG}^{14-16}$. While this has sparked interest in $\mathrm{MDH}$-based materials and devices for THz applications, previous works have delved on utilizing the MDH for THz detection ${ }^{15-19}$, rather than generation.

Previous works have shown that the application of an external magnetic field enhances the $\mathrm{THz}$ emission of several semiconductors ${ }^{20,21}$. We have previously observed in a bare $\mathrm{MDH}$ that the polarity of the applied magnetic field parallel to the surface and normal to the reflection plane dictated the THz enhancement factor ${ }^{22}$, and demonstrated via temperature-dependent THz-time domain spectroscopy (THz-TDS) that the high-field region in the 2DEG is responsible for the $\mathrm{THz}$ emission in a $\mathrm{MDH}^{23}$. This effect was most pronounced in the AlGaAs/GaAs MDH and highest when the external magnetic field was applied parallel to the heterojunction of the MDH. Doing so made the carriers in the MDH mimic the motion of the carriers of a PCA emitter under normal, biased operation ${ }^{24}$.

In this paper, we report on the characteristics of the AlGaAs/GaAs MDH utilized as a PCA with recessed metal contacts, a design we have previously proposed ${ }^{24}$. The MDH PCA emitter, along with standard SI-GaAs and LT-GaAs PCAs, were fabricated by standard lithography techniques and were tested via THz-TDS measurements to understand and compare each of the devices' performance. We show that by exploiting the transport of high-mobility electrons along the 2DEG region, the MDH PCA with recessed contacts shows THz emission amplitude increased by a factor of 7 over that of a SI-GaAs PCA, and roughly by a factor of 1.5 over that of a LT-GaAs PCA. To analyze how the enhanced mobility and reduced scattering would affect the devices, the $\mathrm{THz}$ emission characteristics of the PCA devices using the different substrates, namely SI-GaAs, LT-GaAs, unrecessed $\mathrm{MDH}$ and recessed $\mathrm{MDH}$, were simulated using the Drude-Lorentz model. With its strong THz emission and compact dimensions, the recessed MDH PCA can help pave the way to more efficient, compact, and turn-key $\mathrm{THz}$ spectroscopy solutions.

\section{Photoconductive antenna design and simulation}

Figure 1 shows the cross-section of an AlGaAs/GaAs MDH PCA with surface contacts (Fig. 1a) and recessed contacts (Fig. 1b). The recessed features had an etch depth of $d=187 \mathrm{~nm}$. The PCA pattern used for both was a dipole antenna with a gap of $g=5 \mu \mathrm{m}$. The recessed features could be achieved by selectively etching the layers prior to the deposition of the metal contacts. The proximity of the metal contacts to the 2DEG provides easier access for the electric bias to utilize the 2DEG region of the AlGaAs/GaAs $\mathrm{MDH}$, resulting in a stronger $\mathrm{THz}$ wave emission. Epitaxial growth, lithography and fabrication are discussed in more detail in the Methods.

The mechanism behind the experimental results are supported by numerical simulations of the THz emission using the one-dimensional Drude-Lorentz Model $^{25,26}$. The one-dimensional Drude-Lorentz Model is a simple, yet accurate model of the generation of $\mathrm{THz}$ electromagnetic radiation ${ }^{25,26}$. As a femtosecond optical pulse is made incident onto the photoconductive gap, the photogenerated electron-hole pairs are swept by the applied electrical bias. The transient photocurrent density $j$ is given by

$$
j=e n_{f} v_{h}-e n v_{e}
$$

where $e$ is the electron charge, $n_{f}$ is the free carrier density, $v_{h}$ and $v_{e}$ are the average hole and electron velocities, respectively. The time-dependence of the free carrier density is given by: 


$$
\frac{d n_{f}}{d t}=-\frac{n_{f}}{t_{c}}+G(t)
$$

where $\tau_{c}$ is the carrier capture time and $G(t)$ is the carrier generation rate of the form $n_{0} \exp \left(-t^{2} / p^{2}\right)$ by optical excitation. The acceleration of holes and electrons is given by:

$$
\frac{d v_{h, e}}{d t}=-\frac{v_{h, e}}{\tau_{s}}+\frac{q_{h, e}}{m_{h, e}^{*}} E_{m o l}
$$

where $v_{h, e}$ is the average velocity, $q_{h, e}$ is the charge, $m_{h}^{*}=0.34 m_{e, 0}, m_{e}^{*}=0.067 m_{e, 0}, \tau_{s}$ is the momentum relaxation time given by the Drude relation $\tau_{s}=\mu_{i} m_{i}^{*} / q_{i}$ and $E_{m o l}$ is the local electric field given by:

$$
E_{\text {mol }}=E_{\text {bias }}-\frac{P_{s c}}{\eta \varepsilon}
$$

where $P_{s c}$ is the space-charge polarization created by the carrier separating due to the applied field, $\varepsilon$ is the dielectric constant of the material, and $\eta$ is the geometrical factor of the antenna. For this work, we use $\varepsilon=12.9 \varepsilon_{0}$, which is the dielectric constant of $\mathrm{GaAs}^{27}$.

The time dependence of the space-charge polarization is,

$$
\frac{d P_{s c}}{d t}=-\frac{P_{s c}}{\tau_{r}}+j(t)
$$

where $\tau_{r}$ is the recombination lifetime. Taking the time derivative of Eq. (3), then inserting Eq. (4), the second time-derivative of the velocity $v$ is given by

$$
\frac{d^{2} v_{h, e}(t)}{d t^{2}}=-\frac{1}{\tau_{s}} \frac{d v_{h, e}}{d t}+\frac{q_{h, e}}{m_{h, e}^{*} \eta \varepsilon} \frac{P_{s c}}{\tau_{r}}-\frac{n_{f} e^{2} v_{h, e}}{m_{h, e}^{*} \eta \varepsilon}
$$

Solving both Eqs. (5) and (6) and using Eqs. (1) and (2) will give the photocurrent density j. At far-field, the $\mathrm{THz}$ electric field $E_{\mathrm{THz}}(t)$ is proportional to the time-derivative of the photocurrent density

$$
E_{T H z}(t) \sim \frac{d j}{d t}
$$

The THz wave radiated from the emitter PCA is assumed to reach the PCA detector without any losses. The probe beam generates electron-hole pairs, and the THz electric field incident on the detector PCA sweeps the photocarriers. The current density at the PCA detector is given $b^{28}$ :

$$
j(t)=\int_{-\infty}^{t} \sigma_{s}\left(t-t^{\prime}\right) E_{T H z}\left(t^{\prime}\right) d t^{\prime}
$$

where $\sigma_{s}(t)$ is the transient surface conductivity of the photoconductive substrate of the detector. The transient surface conductivity of the detector was modelled using a LT-GaAs substrate with $\tau_{c, \text { det }}=0.15 \mathrm{ps}, \tau_{s, \text { det }}=40 \mathrm{fs}$. These detector values best replicate the frequency response of the experimental data and are kept as constant PCA detector parameters when simulating the SI-GaAs, LT-GaAs, MDH (Top) and MDH (Recessed) PCA emitters.

The simulation requires physical parameters of the semiconductor substrates, specifically, carrier density $n_{f}$, capture time $\tau_{c}$, scattering time $\tau_{s}$, and recombination time $\tau_{r}$. These parameters have been well-documented for SI-GaAs, while the values for LT-GaAs would depend on the growth temperature. For the MDH samples, Hall mobility measurements were performed to measure the actual carrier density and mobility values. Van der Pauw configuration was utilized by applying indium contacts on top of the MDH and the magnetic field was supplied using a 3 T Lakeshore magnet.

\section{Results and discussion}

Identical dipole-type patterns (gap width $g=5 \mu \mathrm{m}$ ) were fabricated on the surfaces of a SI-GaAs (100) substrate, a LT-GaAs (growth temperature $270{ }^{\circ} \mathrm{C}$ ) substrate, and a MDH substrate; and on a separately prepared piece of the same MDH sample, an antenna was fabricated with the electrical contacts recessed, as described earlier. From here onwards, we refer to the fabricated PCAs as "SI-GaAs", "LT-GaAs", "MDH (Top)" and "MDH (Recessed)". The PCAs were biased at a frequency of $20 \mathrm{kHz}$, and peak-to-peak voltage amplitude of $32 \mathrm{~V}$. The powers of the pump beam and probe beam were both maintained at $9.5 \mathrm{~mW}$, unless otherwise stated. Figure 2a shows the $\mathrm{THz}$ time domain emission spectra from the fabricated PCAs. The generated THz waves were detected using a commercial LT-GaAs dipole-type PCA with a $3.4 \mu \mathrm{m}$ gap. Among the four antennas, the highest THz peak-to-peak amplitude was observed from the MDH (Recessed) PCA, followed by LT-GaAs, MDH (Top) and SI-GaAs PCAs. Between the two MDH PCAs, we find that by recessing the contacts, the bias is able to access the 2DEG region more effectively, and an increase in the drift carrier transport of carriers in the 2DEG resulted in the generation of higher $\mathrm{THz}$ emission.

The dynamic range of the PCAs as a function of THz frequency are shown in Fig. 2b, where each plot was given an appropriate $y$-offset such that the noise floor average coincides with the $\mathrm{y}=0 \mathrm{~dB}$ line (dotted line). The inset shows the $\mathrm{THz}$ power spectra plotted in linear scale, to provide the reader with a visual context of the spectral difference in $\mathrm{THz}$ emission among the devices. The maximum dynamic range for all PCAs are $\sim 60 \mathrm{~dB}$. However, between $0.4 \mathrm{THz}$ and $1.5 \mathrm{THz}$, the dynamic range of the MDH PCAs and LT-GaAs PCAs are higher, by 


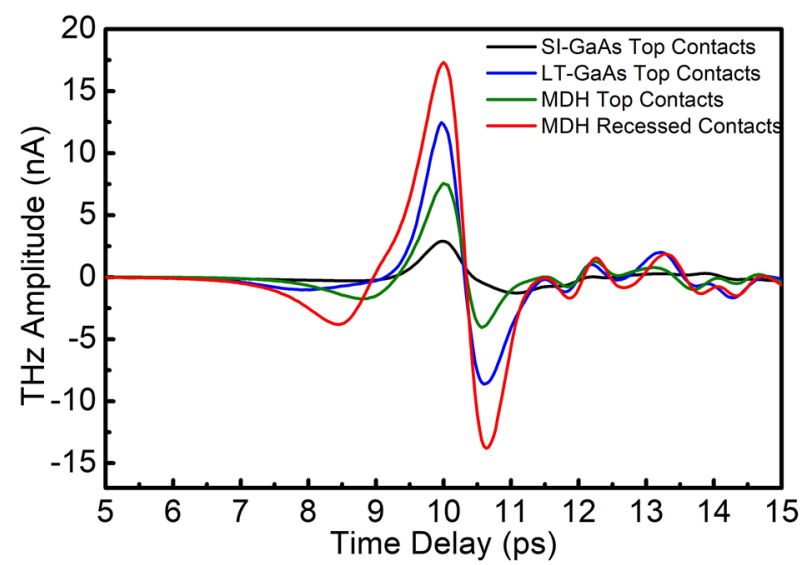

(a)

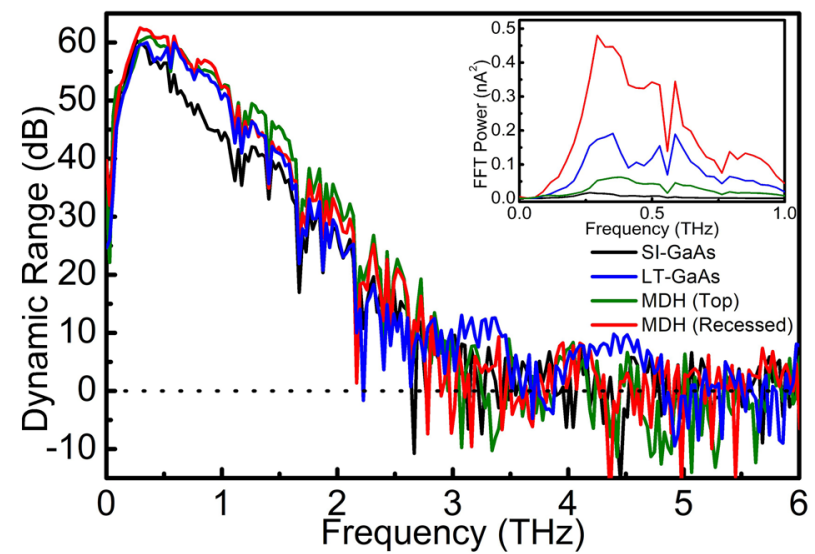

(b)

Figure 2. (a) Measured THz-TDS waveforms and (b) the corresponding normalized dynamic range spectra from the photoconductive antennas. Each plot was given a corresponding $y$-offset in order to align the noise floor average to the $\mathrm{y}=0 \mathrm{~dB}$ line. The inset in $(\mathbf{b})$ is a plot of the frequency power spectra in linear scale.

\begin{tabular}{|l|l|l|l|l|}
\hline PCA & $\begin{array}{l}\text { THz peak to peak } \\
\text { amplitude }(\mathbf{n A})\end{array}$ & Emission bandwidth $(\mathbf{T H z})$ & $\begin{array}{l}\text { Maximum dynamic range } \\
(\mathbf{d B})\end{array}$ & $\begin{array}{l}\text { Integrated THz power } \\
\left(\mathbf{n} \mathbf{A}^{2} \cdot \mathbf{T H z}\right)\end{array}$ \\
\hline SI-GaAs & 4.04 & 3.2 & 60 & $4.5 \times 10^{-3}$ \\
\hline LT-GaAs & 20.92 & 3.2 & 60 & $8.51 \times 10^{-2}$ \\
\hline MDH (Top) & 11.37 & 3.2 & 61 & $2.85 \times 10^{-2}$ \\
\hline MDH (Recessed) & 30.88 & 3.2 & 62 & 0.202 \\
\hline
\end{tabular}

Table 1. PCA performance under $32 \mathrm{~V}_{\mathrm{pp}}, 20 \mathrm{kHz}$ bias and optical pump fluence $3.85 \mathrm{~mJ} / \mathrm{cm}^{2}$.

around $10 \mathrm{~dB}$ at most, compared to the SI-GaAs PCA; and at frequencies higher than $1.5 \mathrm{THz}$, the MDH PCAs have a slightly higher dynamic range $(\sim 5 \mathrm{~dB})$ than the LT-GaAs PCA. The increased density of high velocity carriers participating in the $\mathrm{THz}$ emission process ${ }^{29}$ increases the higher frequency components of the spectra. The performance characteristics are detailed in Table 1 .

The THz waveforms and FFT spectra from photoconductive antenna simulation using the one-dimensional Drude-Lorentz model are shown in Fig. 3 . The parameters used for the carrier density $n_{f}$, capture time $\tau_{c}$, scattering time $\tau_{s}$ and recombination time $\tau_{r}$ used in the simulation are detailed in Table 2. The scattering time was deduced from the experimentally-obtained mobility values using the Drude relation $\tau_{s}=\mu_{i} m_{i}^{*} / q_{i}$ (or vice-versa when the scattering time is known, such as for SI-GaAs and LT-GaAs). The SI-GaAs has a carrier capture time in the order of hundreds of picoseconds, a relatively high scattering time, and high mobility of $>5000 \mathrm{~cm}^{2} /(\mathrm{V} \cdot \mathrm{s})^{30-32}$. The LT-GaAs used in this work was grown at $T_{s}=270^{\circ} \mathrm{C}$ and the presence of defects leads to picosecond carrier lifetime, a low scattering time and a low mobility of $<1000 \mathrm{~cm}^{2} /(\mathrm{V} \cdot \mathrm{s})^{9,33}$. The time-scales of the MDH samples were estimated from literature values based on capacitive measurements or time-resolved measurements ${ }^{34,35}$. For the MDH (Top) structures, the carrier density and mobility were chosen close to actual Hall measurement values while the mobility of the MDH (Recessed) sample was scaled accordingly assuming that the radiated terahertz 


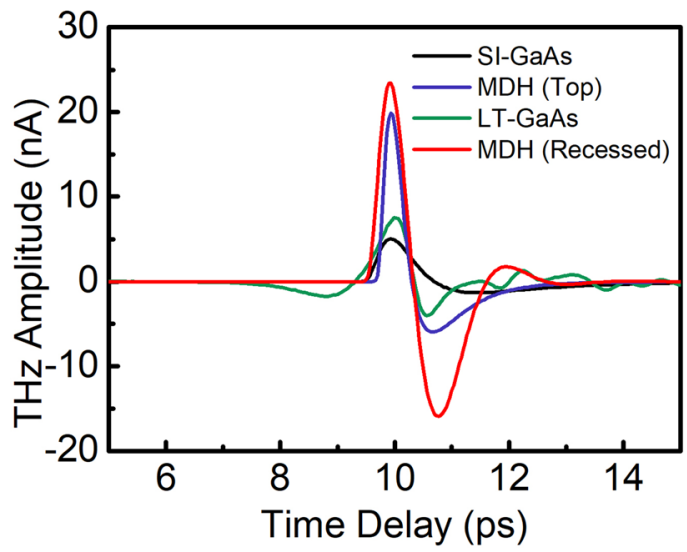

(a)

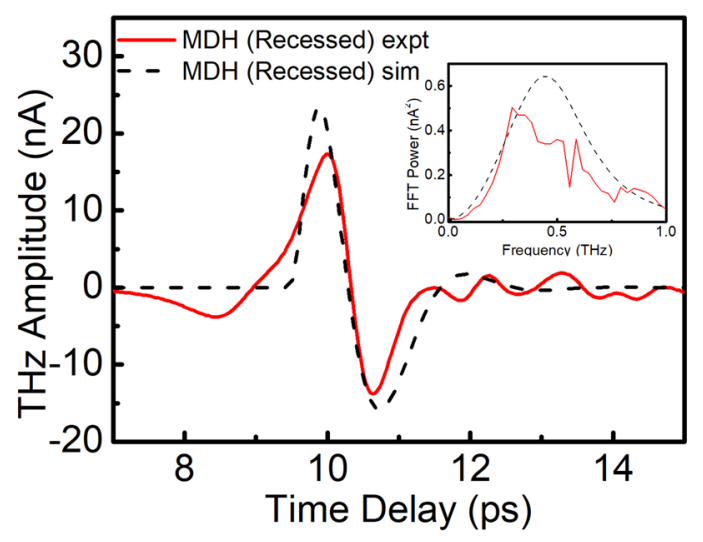

(c)

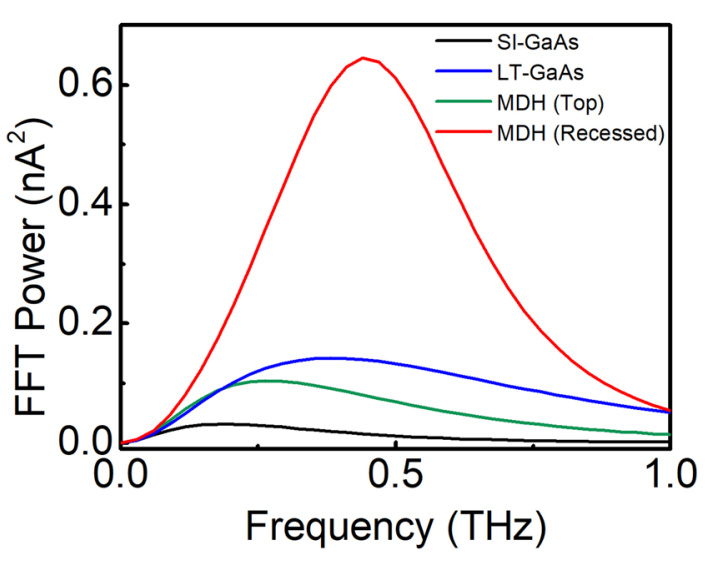

(b)

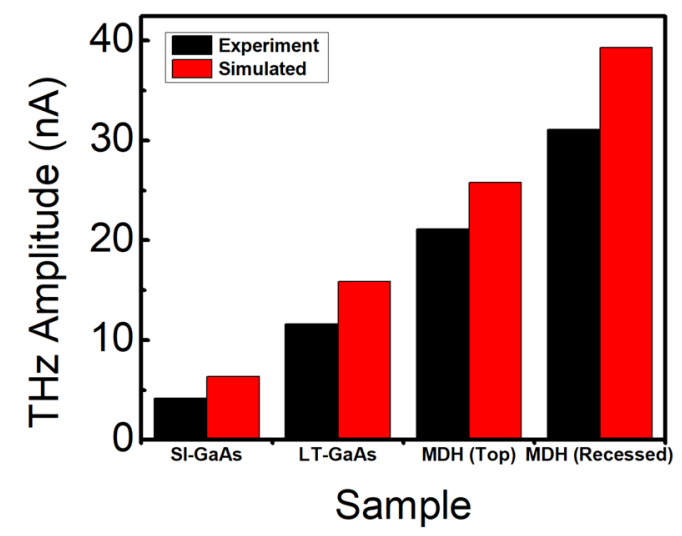

(d)

Figure 3. (a) Simulated THz-TDS waveform and (b) simulated FFT spectra for the samples (c) Representative TDS plot superimposing experimental and simulated results for MDH (Recessed) with inset as FFT spectra (d) Comparison between experimental and simulated peak-to-peak THz amplitude for SI-GaAs, LT-GaAs, MDH (Top) and MDH (Recessed).

\begin{tabular}{|l|l|l|l|l|l|}
\hline PCA & Carrier density, $\boldsymbol{n}_{\boldsymbol{i}}$ & $\begin{array}{l}\text { Trapping time } \\
\text { constant, } \boldsymbol{\tau}_{\boldsymbol{c}}\end{array}$ & $\begin{array}{l}\text { Scattering time } \\
\text { constant, } \boldsymbol{\tau}_{\boldsymbol{s}}\end{array}$ & $\begin{array}{l}\text { Recombination time } \\
\text { constant, } \boldsymbol{\tau}_{\boldsymbol{r}}\end{array}$ & Electron mobility, $\boldsymbol{\mu}$ \\
\hline SI-GaAs & $2.00 \times 10^{16} \mathrm{~cm}^{-336}$ & $100 \mathrm{ps}^{30}$ & $250 \mathrm{fs}^{31}$ & $1 \mathrm{~ns}^{32}$ & $6650 \mathrm{~cm}^{2} \mathrm{~V}^{-1} \mathrm{~s}^{-1 \mathrm{a}}$ \\
\hline LT-GaAs & $1.00 \times 10^{18} \mathrm{~cm}^{-333}$ & $1 \mathrm{ps}^{37}$ & $20 \mathrm{fs}^{38}$ & $100 \mathrm{ps}^{37}$ & $500 \mathrm{~cm}^{2} \mathrm{~V}^{-1} \mathrm{~s}^{-1 \mathrm{a}}$ \\
\hline MDH (Top) & $1.00 \times 10^{17} \mathrm{~cm}^{-3 \mathrm{~b}}$ & $1 \mu \mathrm{s}^{34}$ & $100 \mathrm{fs}^{\mathrm{a}}$ & $1 \mu \mathrm{s}^{35}$ & $2660 \mathrm{~cm}^{2} \mathrm{~V}^{-1} \mathrm{~s}^{-1 \mathrm{~b}}$ \\
\hline MDH (Recessed) & $1.00 \times 10^{17} \mathrm{~cm}^{-3 \mathrm{~b}}$ & $1 \mu \mathrm{s}^{34}$ & $250 \mathrm{fs}^{\mathrm{c}}$ & $1 \mu \mathrm{s}^{35}$ & $6650 \mathrm{~cm}^{2} \mathrm{~V}^{-1} \mathrm{~s}^{-1 \mathrm{c}}$ \\
\hline
\end{tabular}

Table 2. Numerical values used for the one-dimensional Drude-Lorentz PCA simulation. ${ }^{\text {aFrom Drude }}$ relation. ${ }^{b}$ From Hall measurements. ${ }^{c}$ Fitting parameter.

electric field $E_{T H z}$ is directly proportional to the mobility $\mu$. The effective mobility of the carriers contributing to the source current for $\mathrm{THz}$ emission is improved by the direct contact of the metal to the 2DEG region.

The resulting trends in the simulation are in good agreement with the experimentally measured $\mathrm{THz}$ radiation for both the time domain (Figs. 2a, 3a) and the frequency spectra (Figs. 2b inset, 3b). This includes the increased amplitude of higher $\mathrm{THz}$ frequency components with the electron mobility of carriers participating in the $\mathrm{THz}$ generation process. A representative comparison showing the experimental data and simulation for the MDH (Recessed) is shown in Fig. 3c. A deviation between the time-domain waveforms and FFT spectra of the experimental and simulation results is explained by the deformation or renormalization of the $\mathrm{THz}$ waveform due to the antenna response, the frequency-dependent focusing characteristics of the THz optics, and the water vapor absorptions, all of which have been ignored in the simulation.

A comparison between the data and the simulation is presented in a bar graph in Fig. 3d. The differences in $\mathrm{THz}$ emission amplitude between the data and the simulation implies that the etched distance from the surface leaves room for optimization. Nonetheless, the good agreement between the simulation results and 


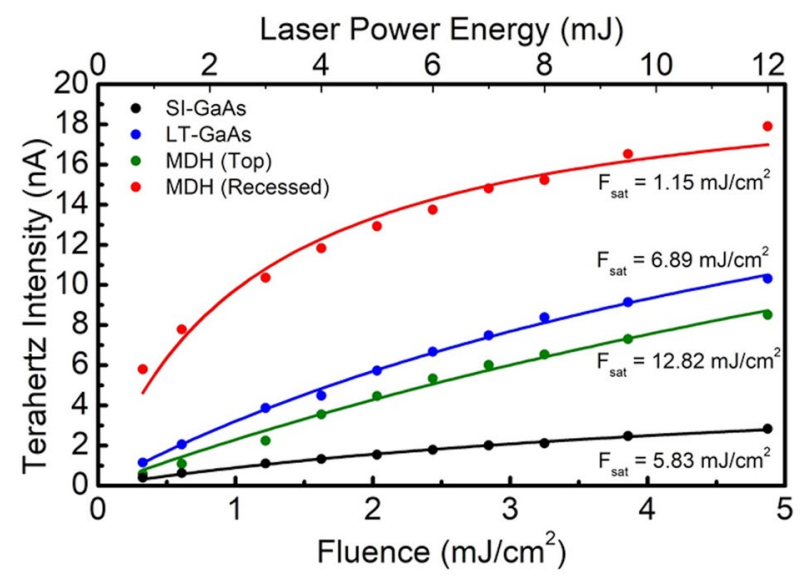

Figure 4. Fluence dependence of the THz peak-to-peak value of the different fabricated PCAs. The solid lines are the saturation curve fitting.

the experimental data shows how the MDH-PCA design effectively utilizes the high-mobility 2DEG region in improving $\mathrm{THz}$ yield.

The dependence of the $\mathrm{THz}$ emission amplitude to the optical fluence was obtained (Fig. 4) by varying the laser power incident on the THz emitter PCAs. At any given pump fluence, the SI-GaAs PCA emits the lowest THz emission amplitude, followed by the MDH (Top) PCA, and lastly, the LT-GaAs PCA. The MDH (Recessed) PCA has the highest THz emission amplitude. Even at the lowest fluence value $\left(<0.5 \mathrm{~mJ} / \mathrm{cm}^{2}\right)$, the THz emission from the MDH (Recessed) PCA was 5 times higher than the THz emission of the LT-GaAs PCA. The saturation fluence $F_{\text {sat }}$ can be calculated from the fits to the equation, $E_{T H z}(F) \approx A\left(F / F_{\text {sat }}\right) /\left(F+F_{\text {sat }}\right)$, where $A$ is the amplitude of the radiated field, and $F$ is the incident beam fluence. The saturation fluence values are $5.83 \mathrm{~mJ} / \mathrm{cm}^{2}$ and $6.89 \mathrm{~mJ} / \mathrm{cm}^{2}$ for the SI-GaAs and LT-GaAs PCAs, respectively. For the MDH (Top) PCA, the $F_{\text {sat }}$ is $12.82 \mathrm{~mJ} /$ $\mathrm{cm}^{2}$. When the contacts are recessed, however, the value for $F_{\text {sat }}$ significantly reduces to $1.15 \mathrm{~mJ} / \mathrm{cm}^{2}$. The saturation of the emitted $\mathrm{THz}$ radiation from PCAs with optical fluence, in general, is attributed to the screening effect that arises from the high photocarrier density ${ }^{39-41}$, and the saturation fluence is inversely proportional to the carrier mobility ${ }^{40}$. With recessed contacts, the applied bias becomes more efficient as it directly accesses the high mobility region; in contrast to when it is applied from the surface. This improved efficiency in the bias conditions outweigh the corresponding detrimental effects of screening.

As a point of reference, LT-GaAs is the most common commercially-available photoconductive material used for emitters because of the ultrashort carrier lifetime due to the high concentration of defects ${ }^{9,42}$. We find that compared to LT-GaAs, the MDH (Recessed) PCA emits a higher THz peak to peak amplitude and has a greater maximum dynamic range, even as they emit at the same THz spectral bandwidth. While LT-GaAs does have a higher saturation fluence at any given moderate fluence value, the efficiency of the MDH (Recessed) PCA is consistently higher in the $<5 \mathrm{~mJ} / \mathrm{cm}^{2}$ fluence range. The MDH (Recessed) PCA would be a good candidate for low laser power applications because of its high THz emission yield. When building THz-TDS spectrometers driven by compact low power fiber lasers, the efficiency of optical-to-THz power is crucial.

In summary, the previously-proposed PCA design was successfully implemented using an n-AlGaAs/GaAs $\mathrm{MDH}$. The MDH was etched to recess the metal for direct contact with the 2DEG region of the MDH. As corroborated by Drude-Lorentz simulation, the influence of the high mobility carriers in the 2DEG was shown to drive the increase in $\mathrm{THz}$ emission. The $\mathrm{MDH}$ recessed contacts have the largest $\mathrm{THz}$ peak-to-peak emission and THz power, as compared to the LT-GaAs and MDH PCAs with top contacts; even as their dynamic and spectral ranges are comparable. The high $\mathrm{THz}$ emission and low saturation fluence of the $\mathrm{MDH}$ recessed contacts offer a feasible solution to THz-TDS systems that are designed to be powered by low power fiber lasers.

\section{Methods}

Figure 5a shows the growth schematics of the n-AlGaAs/GaAs MDH and LT-GaAs. The MDH layer was grown via a RIBER 32P molecular beam epitaxy on an epiready (100)-oriented SI-GaAs substrate. The substrate was first heated in situ at $590{ }^{\circ} \mathrm{C}$ for $10 \mathrm{~min}$ to remove its artificial oxides. The substrate temperature was then raised to $610^{\circ} \mathrm{C}$ to facilitate the growth of a $1.5 \mu \mathrm{m}$ GaAs buffer layer at a growth rate of $1 \mu \mathrm{m} / \mathrm{hr}$. Afterward, this layer was followed by the growth of a $150 \AA$ AlGaAs $(\mathrm{x}=0.2)$ spacer at a growth rate of $\sim 1.2 \mu \mathrm{m} / \mathrm{hr}$. The silicon dopant effusion cell was then opened to facilitate the growth of an $800 \AA \mathrm{n}$-AlGaAs donor layer by using the same growth conditions aside from a nominal doping concentration of $\sim 1 \times 10^{17} \mathrm{~cm}^{-3}$. The growth was then terminated through the growth of a $200 \AA \mathrm{n}$-GaAs cap. The LT-GaAs layer was grown in the same MBE system, on a similar epiready (100) SI-GaAs substrate. The substrate was first heated in situ at $590{ }^{\circ} \mathrm{C}$ for 10 min to remove its artificial oxides. The substrate temperature was then raised to $630^{\circ} \mathrm{C}$ for the growth of a $0.2 \mu \mathrm{m} \mathrm{GaAs}$ buffer. Afterward, the substrate temperature was lowered down to $270{ }^{\circ} \mathrm{C}$ where a $2 \mu \mathrm{m}$ LT-GaAs thin film was grown. The substrate temperature was then raised to $600^{\circ} \mathrm{C}$ in order to anneal the LTG-GaAs layer for $10 \mathrm{~min}$. 
(a)

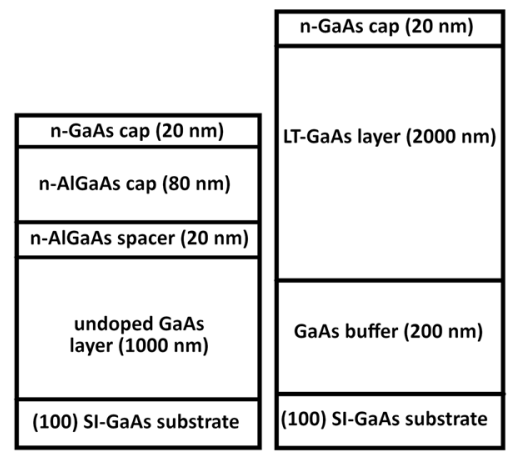

(b)

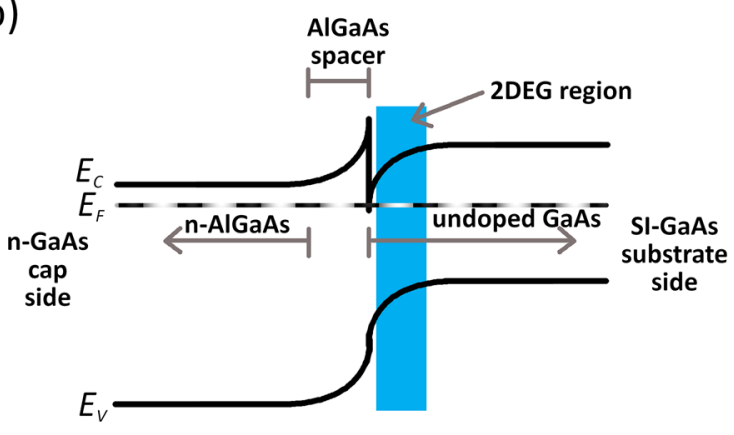

Figure 5. (a) Growth schematic of the n-AlGaAs/GaAs modulation-doped heterostructures and lowtemperature grown $\mathrm{GaAs}$ and $(\mathbf{b})$ band diagram of the $\mathrm{n}-\mathrm{AlGaAs} / \mathrm{GaAs}$ modulation-doped heterostructures. Figures are not to scale.

The growth then terminated through the growth of a $200 \AA$ n-GaAs cap. All of the layers for this sample were grown at a growth rate of $1 \mu \mathrm{m} / \mathrm{h}$.

Figure $5 \mathrm{~b}$ shows the energy band diagram of a typical $\mathrm{MDH}$. At the heterojunction between the highly doped $\mathrm{n}$-GaAs layer and the undoped GaAs, a triangular quantum well is formed due to the alignment of the Fermi energy of the two materials. The MDH structure was originally designed to be used as a HEMT, as conduction electrons in the n-GaAs layer are designed to easily get trapped in the triangular quantum well (transistor channel), where they can move laterally at very low resistance (i.e. high mobility) ${ }^{23,24}$.

All of the samples used, namely two of MDH, LT-GaAs and SI-GaAs, underwent standard degreasing by immersion in trichloroethylene, acetone, and methanol. A MIDAS MDA-400 M mask aligner was used to transfer a dipole PCA structure with a gap $g=5 \mu \mathrm{m}$ onto the surfaces of the LT-GaAs, the SI-GaAs and one of the MDH wafers (to create MDH (Top)). The other MDH substrate (to create the MDH (Recessed) PCA) was also patterned using the same dipole PCA structure, albeit defocused, to obtain a slightly larger pattern than that of the original. This sample was etched in an acid piranha solution consisting of 1:8:80 volumetric ratio of $\mathrm{H}_{2} \mathrm{SO}_{4}: \mathrm{H}_{2} \mathrm{O}_{2}$ : deionized $\mathrm{H}_{2} \mathrm{O}$, which reached a depth of $d=187 \mathrm{~nm}$. After etching, the $\mathrm{MDH}$ (Recessed) substrate was patterned with the same dipole PCA pattern for metallization. AuGe/Ni/Au with nominal thicknesses of 55/15/85 nm were evaporated onto the samples by resistive evaporation and electron beam deposition. After metal lift-off, all of the PCAs were annealed inside a tube furnace at $400{ }^{\circ} \mathrm{C}$ under nitrogen gas-rich environment for $1 \mathrm{~min}$.

The $\mathrm{THz}$ emission characteristics of the samples were measured using a standard THz-TDS spectroscopy setup. The $780 \mathrm{~nm}$ line of a Menlo C-fiber femtosecond fiber laser with pulse duration of $100 \mathrm{fs}$ pulse duration and $100 \mathrm{MHz}$ repetition rate was used. The laser beam was split into pump and probe beams using a beam splitter. The pump beam was used to excite the emitter samples and the probe beams was used to optically gate a commercial 3.4 $\mu \mathrm{m}$ LT-GaAs dipole detector. The pump and probe powers were both maintained at $9.5 \mathrm{~mW}$, unless otherwise stated. The PCA emitters were biased with a $32 \mathrm{~V}$ peak to peak square wave at a frequency of $20 \mathrm{kHz}$.

Received: 8 January 2020; Accepted: 26 October 2020

Published online: 16 November 2020

\section{References}

1. Tonouchi, M. Cutting-edge terahertz technology. Nat. Photon. 1, 97-105 (2007)

2. Yin, M., Tang, S. \& Tong, M. The application of terahertz spectroscopy to liquid petrochemicals detection: a review. Appl. Spectrosc. Rev. 51, 379-396 (2016).

3. Yu, L. et al. The medical application of terahertz technology in non-invasive detection of cells and tissues: opportunities and challenges. RSC Adv. 9, 9354-9363 (2019).

4. Dohi, M. et al. Application of terahertz pulse imaging as PAT tool for non-destructive evaluation of film-coated tablets under different manufacturing conditions. J. Pharm. Biomed. Anal. 119, 104-113 (2016).

5. Petrov, N. V., Kulya, M. S., Tsypkin, A. N., Bespalov, V. G. \& Gorodetsky, A. Application of terahertz pulse time-domain holography for phase imaging. IEEE Trans. Terahertz Sci. Technol. 6, 464-472 (2016).

6. Dong, J. et al. Terahertz frequency-wavelet domain deconvolution for stratigraphic and subsurface investigation of art painting. Opt. Express 24, 26972 (2016).

7. Lepeshov, S., Gorodetsky, A., Krasnok, A., Rafailov, E. \& Belov, P. Enhancement of terahertz photoconductive antenna operation by optical nanoantennas. Laser Photonics Rev. 11, 1600199 (2017).

8. Burford, N. M. \& El-Shenawee, M. O. Review of terahertz photoconductive antenna technology. Opt. Eng. 56, 010901 (2017).

9. Sakai, K. \& Tani, M. Introduction to terahertz pulses. Top. Appl. Phys. 97, 1-30 (2005).

10. Zomega Terahertz Corp. THz System \& Component Catalog.

11. Dingle, R., Störmer, H. L., Gossard, A. C. \& Wiegmann, W. Electron mobilities in modulation-doped semiconductor heterojunction superlattices. Appl. Phys. Lett. 33, 665-667 (1978).

12. Ho, C.-H., Huang, K.-W., Lin, Y.-S. \& Lin, D.-Y. Practical photoluminescence and photoreflectance spectroscopic system for optical characterization of semiconductor devices. Opt. Express 13, 3951 (2005).

13. Ibrahim, S., Ishikawa, H., Nakahara, T. \& Takahashi, R. A novel optoelectronic serial-to-parallel converter for 25-Gbps burst-mode optical packets. Opt. Express 22, 157 (2014). 
14. Dyakonov, M. I. \& Shur, M. S. Two dimensional electronic flute. Appl. Phys. Lett. 67, 1137 (1995).

15. Dyakonov, M. \& Shur, M. Shallow water analogy for a ballistic field effect transistor: new mechanism of plasma wave generation by dc current. Phys. Rev. Lett. 71, 2465-2468 (1993).

16. Dyakonov, M. \& Shur, M. Detection, mixing, and frequency multiplication of terahertz radiation by two-dimensional electronic fluid. IEEE Trans. Electron Devices 43, 380-387 (1996).

17. Meziani, Y. M. et al. Enhancement of sub-terahertz detection by drain-to-source biasing on strained silicon MODFET devices. in J. Phys.: Conf. Ser. 647, 012007 (2015).

18. Shrekenhamer, D. et al. High speed terahertz modulation from metamaterials with embedded high electron mobility transistors. Opt. Express 19, 9968 (2011).

19. Bell, L., Rogers, J., Heyman, J. N., Zimmerman, J. D. \& Gossard, A. C. Terahertz emission by quantum beating in a modulation doped parabolic quantum well. Appl. Phys. Lett. 92, 142108 (2008).

20. Zhang, X. C., Jin, Y., Kingsley, L. E. \& Weiner, M. Influence of electric and magnetic fields on THz radiation. Appl. Phys. Lett. 62, 2477-2479 (1993).

21. Sarukura, N., Ohtake, H., Izumida, S. \& Liu, Z. High average-power THz radiation from femtosecond laser-irradiated InAs in a magnetic field and its elliptical polarization characteristics. J. Appl. Phys. 84, 654-656 (1998).

22. Estacio, E. et al. Transverse magnetic field polarity effects on the terahertz radiation from GaAs/AlGaAs modulation-doped heterostructures with varying AlGaAs spacer-layer thickness. J. Appl. Phys. 104, 073506 (2008).

23. Bardolaza, H. R. et al. Temperature dependence of $\mathrm{THz}$ emission and junction electric field of GaAs-AlGaAs modulation-doped heterostructures with different i-AlGaAs spacer layer thicknesses. J. Mater. Sci. Mater. Electron. 29, 8760-8766 (2018).

24. Bardolaza, H. et al. Efficacy of proposed 2DEG-based photoconductive antenna using magnetic bias-controlled carrier transport. Curr. Appl. Phys. 19, 756-761 (2019).

25. Jepsen, P. U., Jacobsen, R. H. \& Keiding, S. R. Generation and detection of terahertz pulses from biased semiconductor antennas. J. Opt. Soc. Am. B 13, 2424 (1996).

26. Piao, Z., Tani, M. \& Sakai, K. Carrier dynamics and terahertz radiation in photoconductive antennas. Japanese J. Appl. Physics, Part 1 Regul. Pap. Short Notes Rev. Pap. 39, 96-100 (2000).

27. Moore, W. J. \& Holm, R. T. Infrared dielectric constant of gallium arsenide. J. Appl. Phys. 80, 6939-6942 (1996).

28. Lee, Y. S. Principles of terahertz science and technology. Principles of Terahertz Science and Technology (Springer Science \& Business Media, 2009).

29. John Ibanes, J. et al. Terahertz emission from GaAs-AlGaAs core-shell nanowires on $\mathrm{Si}$ (100) substrate: effects of applied magnetic field and excitation wavelength. Appl. Phys. Lett. 102, 063101 (2013).

30. Liu, D. \& Qin, J. Carrier dynamics of terahertz emission from low-temperature-grown GaAs. Appl. Opt. 42, 3678 (2003).

31. Nakajima, M., Takahashi, M. \& Hangyo, M. Strong enhancement of THz radiation intensity from semi-insulating GaAs surfaces at high temperatures. Appl. Phys. Lett. 81, 1462-1464 (2002).

32. Haché, A. et al. Observation of coherently controlled photocurrent in unbiased, bulk GaAs. Phys. Rev. Lett. 78, 306-309 (1997).

33. Mingguang Tuo, Jitao Zhang \& Hao Xin. THz photoconductive antennas. in Developments in Antenna Analysis and Design: Volume 2 73-125 (Institution of Engineering and Technology, 2018). doi:https://doi.org/10.1049/sbew543g_ch3.

34. Canali, C. et al. Trap-related effects in AlGaAs/GaAs HEMTs. IEE proceedings. Part G. Electron. circuits Syst. 138, 104-108 (1991).

35. Gilliland, G. D., Wolford, D. J., Kuech, T. F. \& Bradley, J. A. Long-range, minority-carrier transport in high quality 'surface- free' GaAs/AlGaAs double heterostructures. Appl. Phys. Lett. 59, 216-218 (1991).

36. Okubo, S., Otoki, Y., Watanabe, M., Takahashi, S. \& Kuma, S. Electrical insulation properties of carbon-controlled semi-insulating gaas. Jpn. J. Appl. Phys. 32, 3342-3345 (1993).

37. Khiabani, N., Huang, Y., Shen, Y. C. \& Boyes, S. J. Theoretical modeling of a photoconductive antenna in a terahertz pulsed system. IEEE Trans. Antennas Propag. 61, 1538-1546 (2013).

38. Betko, J., Morvic, M., Novák, J., Förster, A. \& Kordoš, P. Hall mobility analysis in low-temperature-grown molecular-beam epitaxial GaAs. Appl. Phys. Lett. 69, 2563-2565 (1996).

39. Loata, G. C., Thomson, M. D., Löffler, T. \& Roskos, H. G. Radiation field screening in photoconductive antennae studied via pulsed terahertz emission spectroscopy. Appl. Phys. Lett. 91, 232506 (2007).

40. Rodriguez, G. \& Taylor, A. J. Screening of the bias field in terahertz generation from photoconductors. Opt. Lett. 21, 1046-1048 (1996).

41. Tani, M., Matsuura, S., Sakai, K. \& Nakashima, S. Emission characteristics of photoconductive antennas based on low-temperaturegrown GaAs and semi-insulating GaAs. Appl. Opt. 36, 7853-7859 (1997).

42. Kamo, Y., Kitazawa, S., Ohshima, S. \& Hosoda, Y. Highly efficient photoconductive antennas using optimum low-temperaturegrown GaAs layers and Si substrates. Jpn. J. Appl. Phys. 53, 033201 (2014).

\section{Acknowledgements}

This work was sponsored in part by grants from the Department of Science and Technology-Philippine Council for Industry, Energy, and Emerging Technology Research and Development-Grants in Aid (DOST-PCIEERDGIA Project Number 04001) and the DOST-Japan Society for the Promotion of Science Joint Research Program (JSPS) 'Development of the High Performance THz Emitter and Detector and its Application to Material Science'.

\section{Author contributions}

The study was conceived and supervised by E.S.E., where previous studies were led by H.B., J.A., A.D.L.R., N.I.C., et al. The layers for this study were MBE-grown by E.A.P., K.C.G. and G.A.R.C. The PCAs were fabricated by V.D.A.V., N.I.C., M.A.F., J.P.F. at the NIP-UPD. MBE growth and PCA fabrication were supervised by A.A.S. and A.S.S. Photoconductive antenna testing was conducted by E.S.E., A.D.L.R., J.A., V.K.M. at FIR-UF supervised by H.K. and M.T. Subsequent optical testing by A.D.L.R. and H.B. at the NIP-UPD. A.D.L.R. carried out the PCA simulation. The manuscript was prepared by J.A., A.D.L.R., N.I.C., V.D.A.V., E.S.E. and G.A.R.C. All authors have read and approved the contents of the manuscript.

\section{Competing interests}

The authors declare no competing interests.

\section{Additional information}

Correspondence and requests for materials should be addressed to V.D.A.V. or E.S.E.

Reprints and permissions information is available at www.nature.com/reprints. 
Publisher's note Springer Nature remains neutral with regard to jurisdictional claims in published maps and institutional affiliations.

(c) (1) Open Access This article is licensed under a Creative Commons Attribution 4.0 International License, which permits use, sharing, adaptation, distribution and reproduction in any medium or format, as long as you give appropriate credit to the original author(s) and the source, provide a link to the Creative Commons licence, and indicate if changes were made. The images or other third party material in this article are included in the article's Creative Commons licence, unless indicated otherwise in a credit line to the material. If material is not included in the article's Creative Commons licence and your intended use is not permitted by statutory regulation or exceeds the permitted use, you will need to obtain permission directly from the copyright holder. To view a copy of this licence, visit http://creativecommons.org/licenses/by/4.0/.

(c) The Author(s) 2020 